Planetary Systems in the Universe - Observation, Formation and Evolution

Proceedings IAU Symposium No. 202, (C)2004 IAU

Alan Penny, Pawel Artymowicz, Anne-Marie Lagrange, 8 Sara Russell, eds.

\title{
Ground-based exoplanet near-infrared search by imaging and spectroscopy: 3 new companion candidates in TWA
}

\author{
Ralph Neuhäuser \\ MPI extraterrestrische Physik, D-85741 Garching, Germany, and \\ University of Hawaii, Institute for Astronomy, Honolulu, USA
}

Nuria Huélamo

MPI extraterrestrische Physik, D-85741 Garching, Germany

Eike W. Guenther

Thüringer Landessternwarte Tautenburg, D-07778 Tautenburg, Germany

Wolfgang Brandner, João Alves, Fernando Comerón

European Southern Observatory, D-85748 Garching, Germany

Monika G. Petr

MPI für Radioastronomie, Auf dem Hügel 69, D-53121 Bonn, Germany

Jean-Gabriel Cuby

European Southern Observatory, Santiago, Chile

\begin{abstract}
We report first results from our ground-based infrared imaging search for sub-stellar companions (brown dwarfs and giant planets) of young (up to $100 \mathrm{Myrs}$ ) nearby (up to $100 \mathrm{pc}$ ) stars, where companions should be well separated from the central stars and still relatively bright due to ongoing accretion and/or contraction. Our observations are performed mainly with SOFI and SHARP at the ESO $3.5 \mathrm{~m}$ NTT on La Silla (imaging) and with ISAAC at the ESO $8.2 \mathrm{~m}$ Antu (VLT-UT1) on Cerro Paranal (imaging and spectroscopy), all in the $\mathrm{H}$ - and $\mathrm{K}$-bands. Here, we present new companion candidates ${ }^{1}$ around three T Tauri stars (TWA-8 A, RXJ1121.1-3845, and RXJ1121.3-3447 N) in the TW Hya group, which would have been sub-stellar if at the same distance and age as the T Tauri stars, but are found to be background stars by spectroscopy.
\end{abstract}

\section{Introduction}

Sub-stellar companions like brown dwarfs and giant planets are hard to find due to the problem of dynamic range: Faint objects very close to much brighter stars.

\footnotetext{
${ }^{1}$ Based on observations obtained at the European Southern Observatory on Cerro Paranal and La Silla in program 65.L-0144.
} 
All very nearby stars, within $\sim 25 p c$, are $\geq 1$ Gyr old. One way around this problem is to search for sub-stellar companion candidates around very young relatively nearby stars. There are indeed young nearby stars, like GJ $182(\sim 20$ Myrs at $27 \mathrm{pc}$ ), the Tucanae and HorA groups of young zero-age main-sequence stars, the TW Hya association (TWA) of about one dozen pre-main sequence stars isolated from cloud material, and the MBM 12 cloud at $\sim 65 p c$ with several classical and weak-line T Tauri stars.

We have started a direct imaging search for sub-stellar companions around those young nearby stars: Up to $100 \mathrm{Myrs}$ and up to $100 \mathrm{pc}$. Including new nearby stars discovered recently among ROSAT X-ray sources, there are more than 100 such stars known to date. We use the infrared imaging camera SofI at the ESO $3.5 \mathrm{~m}$ NTT on La Silla, the MPE speckle camera SHARP (also used at NTT), the infrared imager and spectrograph ISAAC at the ESO $8.2 \mathrm{~m}$ Antu (VLT-UT1) on Cerro Paranal, and the adaptive optics infrared instrument ALFA on the Calar Alto 3.5m telescope. For companions candidates found in the 1st epoch imaging, we then take a 2nd epoch image one or a few years later for proper motion confirmation and/or an infrared spectrum to confirm or reject their sub-stellar nature.

Five sub-stellar companions have been confirmed so far by both spectroscopy and proper motion: After Gl 229 B, G 196-3 B, and Gl 570 D, the two youngest brown dwarf companions known so far were confirmed by us using spectroscopy and proper motion, namely $\mathrm{CoD}-33^{\circ} 7795 \mathrm{~B}$ in TWA (Neuhäuser et al. 2000b) ${ }^{2}$ and HR 7329 B in Tucanae (Guenther et al. 2001). A giant planet candidate near TWA-7 (Neuhäuser et al. 2000a) has been found to be a background Ktype star in an H-band spectrum taken with ISAAC at the VLT (Neuhäuser et al. 2001).

Here, we report on three more sub-stellar companion candidates found by direct imaging near three other TWA members.

\section{More companion candidates in TWA}

Using the infrared imager Son of Isaac (SofI) at the ESO 3.5m New Technology Telescope (NTT) on La Silla we took images of several TWA members. The observations were made on 2000 May 17 and 19 with $1.0^{\prime \prime}$ to $1.1^{\prime \prime}$ seeing. We used the H-band filter and the small SofI field $\left(0.147^{\prime \prime} / \mathrm{pix}\right)$ with a total exposure time of $10 \mathrm{~min}$ per target $(460 \times 1.3 \mathrm{sec}$ in auto-jitter mode). Darks, flats, and standard stars were taken in the same night, and we performed standard data reduction with eclipse $e^{3}$ and MIDAS. We found new companion candidates around TWA-8 (a TWA T Tauri star found by Webb et al. 1999), RXJ1121.13845 (=GSC 7739 2190), and RXJ1121.3-3447 (=GSC 7210 1352), originally found as new $\mathrm{T}$ Tauri stars by W. Hoff ( $\mathrm{PhD}$ thesis) and published by Sterzik et al. (1999), data are listed in table 1.

\footnotetext{
${ }^{2}$ Schneider et al. (2001) confirmed the M8.5-M9 spectral type reported by Neuhäuser et al. (2001) with an HST/STIS spectrum with a smaller wavelength range.

${ }^{3}$ www.eso.org/eclipse
} 
Table 1: Three new companion candidates $(=\mathrm{c} / \mathrm{c})$ in TWA

\begin{tabular}{lcc|ccc|c}
\hline \multirow{2}{*}{$\begin{array}{l}\text { Primary star } \\
\text { Name }\end{array}$} & Sp.Type & $\mathrm{H}[\mathrm{mag}]$ & $\Delta \alpha$ & $\Delta \delta$ & sep ["] & $\mathrm{H}[\mathrm{mag}]$ \\
\hline TWA-8 & M2,M5 & $7.8,9.4$ & $5.6^{\prime \prime} \mathrm{E}$ & $8.3^{\prime \prime} \mathrm{N}$ & 10.0 & 15.5 \\
RXJ1121.1-3845 & M2 & 8.4 & $6.0^{\prime \prime} \mathrm{W}$ & $2.6^{\prime \prime} \mathrm{S}$ & 6.5 & 16.5 \\
RXJ1121.3-3447 & M1,M2 & $7.7,7.8$ & $5.4^{\prime \prime} \mathrm{W}$ & $9.1^{\prime \prime} \mathrm{N}$ & 10.6 & 16.8 \\
\hline
\end{tabular}

Remarks: (*) TWA-8 and RXJ1121.2-3446 are binary stars, the separations given are measured from the $\mathrm{c} / \mathrm{c}$ to the primary (northern) star. All mags $\pm 0.1 \mathrm{mag}$, sep $\pm 0.1^{\prime \prime}$. The companion candidate to RXJ1121.1-3845 was found also by D. Trilling \& R. Jayawardhana using the cold coronograph CoCo at the NASA IRTF (priv. com.).

If the companion candidates would be bound, i.e. at $\sim 55 p c$ and $\sim 10$ Myrs as TWA, their magnitudes would correspond to objects with only a few Jupiter masses, according to different sub-stellar tracks and isochrones, i.e. they would be sub-stellar. We took follow-up H-band spectra using ISAAC at Antu (VLTUT1) and found that all three companion candidates are unrelated background stars (to be reported in more detail later). No companion candidates were found within 15" around RXJ1109.7-3907 (PPM 288568, H=8.8 mag), the other new TWA T Tauri found by Hoff and Sterzik et al., down to $H \simeq 17$ mag.

\section{Summary}

Our observations of TWA-7 and TWA-5 (as well as those presented here), all performed with ground-based present-day technology, show that objects as faint as expected for young nearby giant planets located very close (few arc sec) to much brighter stars $(\Delta \mathrm{mag} \simeq 10 \mathrm{mag})$ can be detected already. Once such a planetary companion candidate is confirmed, we can probe its atmosphere by spectroscopy.

\section{References}

Guenther, E.W., Neuhäuser, R., Brandner, W., et al. 2001, A\&A, 365, 514

Neuhäuser, R., Brandner, W., Eckart, A., et al. 2000a, A\&A, 354, L9

Neuhäuser, R., Guenther, E.W., Petr, M.G., et al. 2000b, A\&A, 360, L39

Neuhäuser, R., Guenther, E.W., Brandner, W., et al. 2001, in ASP Conf. Ser., From darkness to light, ed. Montmerle T. \& Andre P., in press

Schneider, G., Lowrance, P.J., Becklin, E.E., et al. 2001, in Lecture Notes in Physics, Springer, in press

Sterzik M.F., Alcalá J.M., Covino E., \& Petr M.G. 1999, A\&A, 346, L41

Webb, R.A., Zuckerman, B., Platais, I., et al. 1999, ApJ, 512, L63 